\title{
An Adaptive Base Point Algorithm for the Retrieval of Aerosol Microphysical Properties
}

\author{
L. Osterloh ${ }^{*, 1}$, C. Böckmann ${ }^{1}$, R.E. Mamouri ${ }^{2}$ and A. Papayannis ${ }^{2}$ \\ ${ }^{1}$ Institute of Mathematics, University of Potsdam, Am Neuen Palais 10, D-14469 Potsdam, Germany \\ ${ }^{2}$ National Technical University of Athens, Laser Remote Sensing Laboratory, Heroon Polytechniou 9, Zografou, 15780 \\ Athens, Greece
}

\begin{abstract}
We propose a new iterative algorithm for the retrieval of the microphysical properties of stratospheric and tropospheric aerosols from multiwavelength lidar data. We consider the basic equation as an ill-posed problem and solve the system derived from spline collocation via a Padé iteration. The algorithm takes special care of the fact that the reconstruction of the distribution via spline collocation is very sensitive to the choice of base points of the chosen spline basis. The algorithm makes use of this fact by changing the base points used for the spline collocation at certain iteration steps. In addition, the effects of projection to ensure a nonnegative solution are examined. We tested how well this and other algorithms are suitable for retrieving the complex refractive index of the particles as well. We also examine whether the algorithm is capable of distinguishing between different, very small imaginary parts of the refractive index, which is often a main problem in practice. Finally, the algorithm is applied to real multiwavelength Raman lidar data and our results are partially validated by the thermodynamic chemical model Isorropia II.
\end{abstract}

Keywords: Aerosol particles, lidar, inverse problem, microphysical parameters.

\section{INTRODUCTION}

Aerosol particles in the Earth's atmosphere are having a wide range of influences on local and global climate and weather, such as on clouds and precipitation. They are important to understanding the chemical processes happening in the troposphere and the stratosphere, see [1]. Also, the direct and indirect effects of aerosols are the two largest contributions to the total uncertainty of the radiative forcing, which roughly describes the difference between incoming and outgoing energy of the tropopause, and thus can be used in researching global climate change.

There exist several methods to measure the concentration and attributes of aerosol particles, like the refractive index to estimate the single scattering albedo, or for instance aerosol size. One such method is lidar (light detection and ranging). In this paper, we will discuss a new algorithm to extract information about the distribution of the particles from multiwavelength lidar data, or more specifically, from extinction and backscatter coefficients gained from the respective profiles.

For multiple wavelength lidar we can obtain the following equation, in this case for number distribution of the particles

$$
\Gamma(\lambda)=\int_{r_{\min }}^{r_{\max }} \pi r^{2} Q_{\pi / \mathrm{ext}}(r, \lambda, m) n(r) \mathrm{d} r
$$

\footnotetext{
*Address correspondence to this author at the Institute of Mathematics, University of Potsdam, Am Neuen Palais 10, D-14469 Potsdam, Germany; Tel: +49 331977 1467; Fax: +49 331977 1001;

E-mail: osterloh@uni-potsdam.de
}

Note that these functions $Q$ are the Mie kernel functions for spherical particles for backscatter $(\pi)$ and extinction (ext) wavelengths, respectively (see [2]). Here, $\Gamma$ is the backscatter or extinction coefficient, $m$ the complex refractive index, $\lambda$ the wavelength and $n(r)$ the particle size distribution. The radii $r_{\min }$ and $r_{\max }$ are sensible lower and upper bounds for the size of the particles.

In common measurement setups, we expect a lidar setup consisting of 3 backscatter wavelengths (at 355, 532 and $1064 \mathrm{~nm}$ ) and 2 extinction wavelengths (at 355 and $532 \mathrm{~nm}$ ).

Equation (1) is an integral equation of first Fredholm kind, thus, an ill-posed problem requiring regularization (see [3]). As can be seen from equation (1), the kernel function of this integral equation depends on the radius $r$ (which is the integration variable), the wavelength $\lambda$ and the refractive index $m$.

Much research has been done already in the area of solving this inverse equation. One approach that has been followed in [4,5] makes use of spline collocation and truncated singular decomposition. Also, to solve the nonlinear problem with unknown refractive index, the retrieval of the refractive index is handled by calculating solutions on a predefined grid and manually picking out solutions from that grid by criteria like the residual error. This is also done in the algorithm from [6], except that a Tikhonov method is used for the regularization here. Moreover, [6] employs a two-dimensional regularization approach which reduces extensive data postprocessing procedures. Also, there is lots of research into the questions on how special a priori information on the solution can be used to improve the results. In [7], for instance, a special 
algorithm for the retrieval of bi-modal distributions is proposed. Another example can be found in [8], which takes special care to incorporate a priori information on the solution in the form of a nonnegativity constraint. Also, new mathematical methods have been considered, like for instance the maximum entropy method in [9] or Runge-Kutta type iteration methods in [10]. In this paper, we propose a novel method of finding a general formula for retrieving the aerosol microphsyical properties from inversion of multiwavelength lidar data, which can be used in a wide area of applications, not specializing on certain cases where lots of a priori information about the particles is already given.

Assuming the refractive index to be unknown (and thus, treating it as a variable for the equation) leads to a nonlinear ill-posed problem; we will not look at this approach. Instead, it is possible to look at the linear problem with fixed refractive index for each point on a predefined refractive index grid containing all viable possibilities, see [5], which will also be the method we will use here.

In this paper, we will focus solely on the retrieval of the volume distribution and refractive index, and the properties derived from it, like the effective radius or the volume concentration; of course, there exist several other problems on the chain from lidar signal data to microphysical properties, like obtaining the extinction and backscatter profiles from the preprocessed signals (see $[11,12]$ ), the recognition of homogenous aerosol layers in these profiles, see [6], or the computational complexity of the whole problem (see [13]). The EARLINET measurements started in May 2000 and are still ongoing; up to now, the EARLINET database represents the largest database for the aerosol distribution on a continental scale. It contains more than 20000 aerosol profiles in terms of extinction, backscatter and lidar ratio, where the lidar ratio data has been retrieved from simultaneous and independent lidar measurements of aerosol extinction and backscatter, see [14]. Currently, the EARLINET network is running the EARLINET-ASOS 5year EU project, whose main objective is to provide accurate, well-defined, and easily accessible data products for use in science and environmental services. In particular, the optimization of the algorithms for the retrieval of the aerosol optical and microphysical properties is a crucial activity, with the main objective of providing a processing chain for the evaluation of lidar data, from raw signals to final products, see [15].

\section{REGULARIZATION}

The problem is, as mentioned, an ill-posed Fredholm integral equation of first kind. Thus, we regard the equation

$y(s)=A x=\int_{a}^{b} k(s, t) x(t) \mathrm{d} t$.

In this case, we assume $A$ to be a compact operator between separable Hilbert spaces $X$ and $Y$. This is always the case for $t \in[a, b]$ and $s \in[c, d]$, thus $X=L^{2}[a, b]$ and $Y=L^{2}[c, d]$ and $k \in L^{2}([c, d] \times[a, b])$. Note here that $L^{2}[a, b]$ denotes the space of square-integrable functions on the interval $[a, b]$, and $L^{2}([c, d] \times[a, b])$ on the twodimensional interval $[c, d] \times[a, b]$ accordingly. This type of operator is always ill-posed in the sense of the Hadamard definition (see [3]).

We cannot just invert the operator $A$ to get $A^{-1}$ for the simple reason that $A^{-1}$ usually does not exist. We achieve existence and uniqueness by looking for the minimum-norm solution of $\inf _{x \in X}\|A x-y\|$, which exists roughly spoken for all regular cases, and can be obtained via the Moore-Penrose inverse and the normal equations

$A^{*} A x=A^{*} y$,

thus

$x=A^{+} y:=\left(A^{*} A\right)^{-1} A^{*} y=\sum_{j=1}^{\infty} \frac{\left\langle y, v_{j}\right\rangle}{\sigma_{j}} u_{j}$.

Here, $\left\langle y, v_{j}\right\rangle$ denotes the inner product and $A^{*}$ denotes the adjoint operator of $A$. We know that such a compact operator has a singular system $\left\{\left(\sigma_{j} ; u_{j}, v_{j}\right)\right\}$, with the $\sigma_{j}$ being the singular values and the $u_{j}, v_{j}$ being the singular vectors. Usually, the singular values are ordered in descending order. The problem here that produces the instability is the fact that the singular values cluster at zero, thus amplifying the high frequencies which are usually present in noisy data.

So, the minimum-norm solution usually has no value at all; regularization is needed. Well-known and examined regularization techniques include the Truncated Singular Value Decomposition (TSVD), see [4], which just cuts off all the frequencies below a certain threshold $v$, thus

$R_{\mathrm{TSVD}} y=\sum_{j=1}^{n} \frac{\left\langle y, v_{j}\right\rangle}{\sigma_{j}} u_{j}$

with $\sigma_{n} \geq v$ and $\sigma_{n+1}<v$. Another technique that is widely used is the Tikhonov-Phillips Regularization (TPR), which shifts the spectrum of the operator (see $[3,6,7]$ ), thus

$R_{\mathrm{TPR}} y=\sum_{j=1}^{\infty} \frac{\left\langle y, v_{j}\right\rangle}{\sigma_{j}+\delta^{2}} u_{j}$

for a certain $\delta \in \mathbb{R}$. Yet another method often used is the iterative Landweber method; the iteration equation is

$x_{i+1}=\left(I-\tau A^{*} A\right) x_{i}+\tau A^{*} y$,

where $\tau$ is the relaxation parameter and acts as a sort of step-length for the iteration. Here, the number of iteration steps performed until the iteration is stopped (denoted $k^{*}$ ) acts as the regularization parameter.

This iteration converges to $A^{+} y$ for $\tau \in\left(0,2 /\|A\|^{2}\right)$.

In all cases, the question remains on how to choose the regularization parameter (in our examples, $v, \delta$ and $k^{*}$ ); this is done by parameter choice rules or stopping rules. The problem about the Landweber method (7) is its slow speed of convergence. From there, alternative iteration methods were developed which converge to a specified tolerance much 
faster because the constraints on the relaxation parameter $\tau$ can be dropped. The method we are going to present here is of preconditioner type; that is, our iteration equation becomes

$x_{i+1}=\left(I-\tau T A^{*} A\right) x_{i}+\tau T A^{*} y$,

where $T$ is the preconditioning operator. The methods we are looking at in this paper are called Padé iterations (or, closely related, the Runge-Kutta iterations) and have been examined in $[10,16,17]$. These methods are derived from Padé approximations to the exponential function; indeed, it can be shown that using Showalter's method, solving the ordinary differential equation

$\dot{x}+A^{*} A x=A^{*} y$

with $x(0)=0$ using the explicit Euler method results in the Landweber iteration, with the steplength of the Euler method corresponding to the relaxation parameter. Using other Runge-Kutta schemes, or Padé approximations to the exponential function, results in other regularization techniques; furthermore, for certain types of these iterations, the constraints on the relaxation parameter can be dropped and theoretically an arbitrary speed of convergence can be achieved. One of those methods is the $(2,1)$-Padé iteration, see [16], denoted by the operator $P^{(2,1)}$; the preconditioner here is defined by the polynomial

$t(s)=\frac{1+s / 3}{1-2 s / 3+s^{2} / 6}$,

which results in the iteration equation

$$
\begin{aligned}
& P^{(2,1)} x_{i}:=x_{i+1}=x_{i}+ \\
& \tau \sum_{j=0}^{\infty} \frac{1+\tau \frac{\sigma_{j}^{2}}{3}}{1-2 \tau \frac{\sigma_{j}^{2}}{3}+\tau^{2} \frac{\sigma_{j}^{4}}{6}}\left\langle A^{*} y-A^{*} A x_{i}, u_{j}\right\rangle u_{j} .
\end{aligned}
$$

Note here that Padé is mainly chosen here for the massive speedup that can be obtained from using it, as there is no restriction on the relaxation parameter; the quality of the solution of a pure Padé iteration is nearly exactly the same as for the Landweber iteration. Finally, to get a regularization in the mathematical sense, we also need a stopping rule that stops the iteration after a finite number of steps, when the reconstruction fulfills certain properties. Here, we will use Morozov's discrepancy principle (see [3]), which stops at the index $k^{*}$ with $k^{*}=\min _{k \in \mathbb{N}}\left\|y-A x_{k}\right\| \leq \gamma \varepsilon$, with $\gamma>1$ and $\left\|y-y^{*}\right\| \leq \varepsilon$ for $y^{*}$ the unperturbed right-hand side, $y^{*}=A x^{*}$. Note that, for numerical stability reasons, we are reconstructing the particle volume distribution instead of the size distribution; however, these two distributions can be directly calculated from each other.

Since we are trying to reconstruct the particle volume distribution, it is obvious that our true solutions are functions $x^{*}(t)$ with $x^{*}(t) \geq 0$ for $t \in\left[r_{\min }, r_{\max }\right]$ (i.e., nonnegative functions). This condition on the solution, however, will usually not be fulfilled for a reconstructed solution $x(t)$. It is possible to include the information of nonnegativity as a priori information on the solution, though. We will do this by performing an orthogonal projection on the set of nonnegative functions after each iteration step; thus, the iteration equation (11) becomes

$x_{i+1}=\mathcal{P}_{L_{+}^{2}\left[r_{\min }, r_{\max }\right]} P^{(2,1)} x_{i}$,

where $\mathcal{P}_{L_{+}^{2}\left[r_{\text {min }}, r_{\text {max }}\right]}$ denotes the orthogonal projector onto the set of nonnegative square-integrable functions. See Fig. (1) for the effects of projection on our regularized solution. Note that the plot shown in Fig. (1) is the volume distribution $v(r)$ of the particles. The volume concentration $v_{t}$ is usually given for aerosols in $\mu \mathrm{m}^{3} / \mathrm{cm}^{3}$. As the volume distribution is defined as $\frac{\mathrm{d} v_{t}}{\mathrm{~d} r}$, the resulting unit for $v(r)$ is the given $\mu \mathrm{m}^{3} /\left(\mathrm{cm}^{3} \mu \mathrm{m}\right)$. This is the unit often used in the context of aerosol volume concentration, for instance in [6,7]. In that figure, see the reconstruction of a mono-modal log-normal distribution; the definition of the multi-modal log-normal distribution,

$n(r)=\sum_{j=1}^{M} \frac{N_{t, j}}{\sqrt{2 \pi} r \ln \bar{\sigma}_{j}} \exp \left(\frac{\left(\ln r-\ln r_{\text {med }, j}\right)^{2}}{-2\left(\ln \bar{\sigma}_{j}\right)^{2}}\right)$,

where $N_{t, j}$ is the total number concentration, $\bar{\sigma}_{j}$ the geometric standard deviation and $r_{\text {med }, j}$ the median radius of the mode $j$. The number of modes is described by $M$, so in the mono-modal case we have $M=1$, and we can omit the index $j$.

\section{DISCRETIZATION}

For the measurement case, we look at the semi-discrete problem $A: L^{2}\left[r_{\min }, r_{\max }\right] \rightarrow \mathbb{R}^{n}$, which maps from the infinite-dimensional space of square-integrable functions on $\left[r_{\min }, r_{\max }\right]$ to the finite-dimensional space of real vectors with $n$ entries. This is due to the fact that in practice we only have a finite number of data points (in lidar case, backscatter and extinction coefficients at certain wavelengths). Now, for actually calculating solutions, we also need to discretize in a fitting way. It has been shown that discretization by projection in itself is already a regularization, with the coarseness of the discretization acting as a sort of regularization parameter. Several methods can be used for discretizing the space $L^{2}\left[r_{\min }, r_{\max }\right]$, such as standard quadrature approaches or Galerkin discretization. In this paper, we will make use of spline collocation using Bsplines, thus projecting to a finite-dimensional space. For more information on B splines than the quick introduction here, please refer to [18].

Assume our data is available for $n$ wavelengths, $\lambda_{1}<\lambda_{2}<\ldots<\lambda_{n}$. Let us project the space $L^{2}\left[r_{\min }, r_{\max }\right]$ to dimension $l$; for spline collocation, that means we have a spline basis with $l$ elements for the space. Let us choose a 

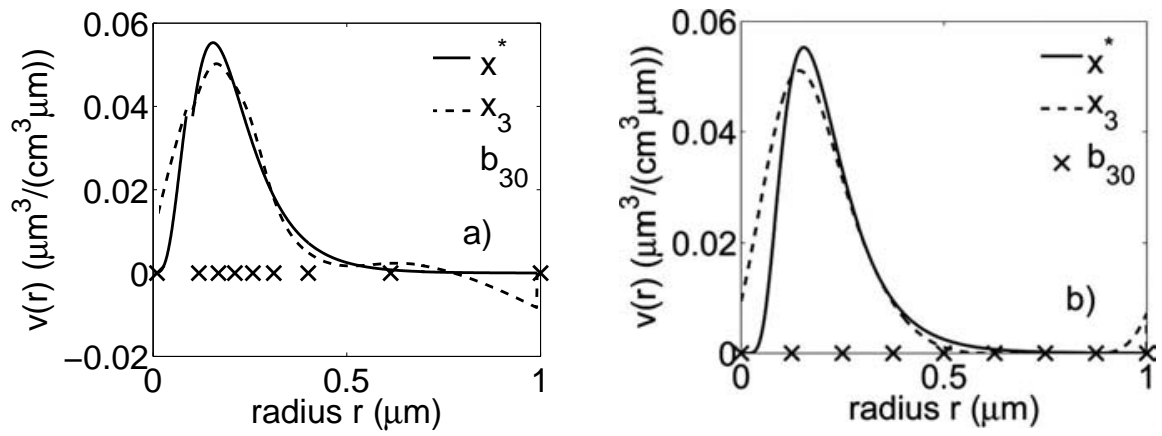

Fig. (1). Reconstruction of a monomodal distribution with $r_{\text {med }}=0.1, \bar{\sigma}=1.6$ and $N_{t}=1$. On the left, the solution is reconstructed without projection, on the right, without sliding the base points, but with projection. The solid line marks the true solution $x^{*}$, the dashed line the reconstruction after thirty iterations $x_{30}$. The $\times$ symbols mark the base points at that iteration.

vector of base points $b=\left(b_{1}, \ldots, b_{n}\right)$ and a degree $d$ for the $\mathrm{B}$ splines. In this case, we have $l=n+d-2$. The actual splines of degree $d$ can be calculated by the recursion defined by

$N_{i, 1}(r)=\chi_{\left[b_{i}, b_{i+1}\right]}(r)$

and

$N_{i, d}(r)=\frac{r-b_{i}}{b_{i+d-1}-b_{i}} N_{i, d-1}(r)+\frac{b_{i+d}-r}{b_{i+d}-b_{i+1}} N_{i+1, d-1}(r)$.

We will denote this basis of B splines of degree $d$ by $\left\{\phi_{1}, \phi_{2}, \ldots, \phi_{l}\right\}$. We now have to solve the equation

$A x=y\left(\lambda_{i}\right)$,

where the matrix $A \in \mathbb{R}^{l, n}$ is computed by

$A_{i j}=\int_{r_{\min }}^{r_{\max }} \pi r^{2} Q_{\pi / e x t}\left(r, \lambda_{i}, m\right) \phi_{j}(r) \mathrm{d} r$.

We will use the $(2,1)$-Padé iteration to solve (15). As a stopping rule, the usual discrepancy principle will be applied, which stops the iteration at the index $k^{*}$ with

$\left\|y-A x_{k}{ }_{k}\right\| \leq \gamma \varepsilon<\left\|y-A x_{k}\right\|$

for all $k=0,1, \ldots, k^{*}-1$ and some $\gamma>1$. The data error is denoted by $\varepsilon$ and fulfills the property $\left\|y-y^{*}\right\| \leq \varepsilon$. To ensure regularization properties of this method in the mathematical sense, we just turn off the projection after a certain number of projected iterations $p$ and restart the iteration with the last iterate as starting value and without projection, thus our iteration equation becomes

$x_{i+1}=\mathcal{P}_{L_{+}^{2}\left[r_{\min }, r_{\max }\right]} P^{(2,1)} x_{i}$

for $i<p$ and

$x_{i+1}=P^{(2,1)} x_{i}$

for $i \geq p$, theoretically rendering the method a normal Padé iteration equation (without projection), with a starting value calculated by a certain algorithm (a projected iteration). The main difference here to the algorithm presented in [10] is the projection in the first $p$ iteration steps. For results obtained by this algorithm, take a look at the result sections, 5 and 6 .

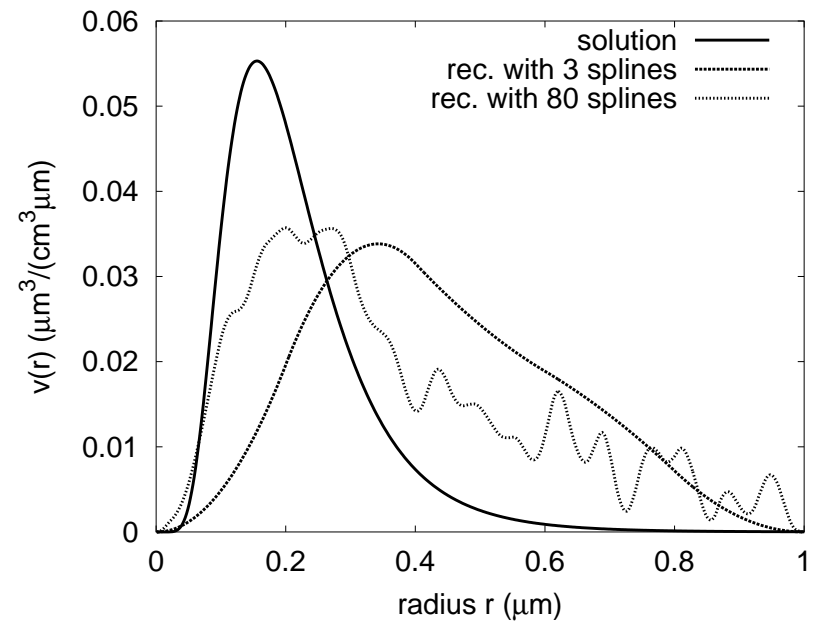

Fig. (2). Reconstruction of a monomodal distribution with $r_{\text {med }}=0.1, \bar{\sigma}=1.6$ and $N_{t}=1$. The solid line is the true solution, the dashed line the reconstruction with 80 splines and the dotted line with 3 splines.

As mentioned above, discretization is in itself already a regularization. It is well known though that the regularizing effect of discretization is small, so additional regularization is needed in most cases except when the problem is very mildly ill-posed. This also directly pertains to the question on how to choose the number of base points (or splines, as these numbers are directly dependent on each other). While choosing far too few or too many base points results in the known effects of over- or underregularization (see Fig. 2), all values that are less extreme are expected to lead to sensible results. In Fig. (2), there was no data error added. Still, it is possible to see how very extreme choices can already prevent proper regularization. Of course, a completely general conclusion as the number of splines cannot be made because it is always dependent on the input data, too. What we can roughly say is that any number in the 8-20 range is acceptable for the kind of input data we usually expect in our lidar environment. If the number of splines is chosen in that area, the methodology works in all cases we have tried so far. Of course, as always with ill-posed problems, large error levels can make the results unfeasible; again, this cannot be 
generalized because it heavily depends on the input data itself.

\section{ADAPTIVE CHOICE OF BASE POINTS}

In the following section, a newly developed algorithm will be compared to two other algorithms. The Padé algorithm without base point adaptation and without projection from [10] will be referred to as algorithm 2, and the hybrid regularization method (TSVD) from [4] equipped with the discrepancy principle as algorithm 1 .

There is a strong connection between the choice of base points and the quality of the reconstruction of the volume distribution. To take that into consideration, the authors propose an algorithm that adapts the base points according to certain parameters. Indeed, it is easy to see how the solution is strongly smoothed out in areas with only a few base points, and sharp features require lots of base points in their vicinity to be reconstructed accurately. To respect that fact, our idea is to slide the base points according to some scheme. Thus, we propose to make the base points of the splines variable, sliding them towards the points that have more weight in the function. This can be done a certain number of times, always projecting the iteration in between. Of course, the iteration operator $P^{(2,1)}$ has to be recalculated after every base point change. Denote the vector of base points in iteration step $k$ with $b_{k}$. After we get a final set of base points, we restart the Padé iteration with our last projected iterate as starting value, and stop with the discrepancy principle, thus rendering the algorithm a regularization in the mathematical sense. Indeed, in our experiments, the discrepancy principle always stopped after the first iteration, hinting at the fact that the starting value is already close to the solution. Let us now formalize this algorithm, further referred to as algorithm 3 .

\subsection{Adaptive Base point Padé Algorithm}

1. Choose an equidistant grid of base points $b_{0}$ with $b_{0} \in \mathbb{R}^{n}$ and a degree $d$ for the $\mathrm{B}$ splines. Calculate $P^{(2,1)}$. Set index $i=0$.

2. Perform $k_{1}$ projected Padé iterations, see equation (12).

3. After $k_{1}$ iterations, compute volume distribution $v_{i}(r)$.

from spline coefficients $x_{i}$ as a linear combination with the splines. Equidistantly discretize $v_{i}(r)$ to a vector $v_{i} \in \mathbb{R}^{P}$. Choose new base points $\left(b_{i}\right)_{j}, j=1, \ldots, n$ according to this formula:

$\left(b_{i}\right)_{j}=\min _{N \in \mathbb{N}}\left\{\sum_{p=1}^{N}\left(v_{k}\right)_{p} \geq w_{j} \sum_{p=1}^{P}\left(v_{k}\right)_{p}\right\}$,

where $w \in \mathbb{R}^{n}$ with $r_{\min }=w_{1}<w_{2}<w_{3}<\ldots<w_{n}=r_{\max }$. With the vector of new base points $\left(b_{i}\right)$, recalculate $P^{(2,1)}$. Calculate the new $x_{i}$ as the best approximation to the old $x_{i}$ in the new spline space.

4. Repeat steps 2 and $3 l$ times.
5. Perform $k_{2}$ projected Padé iterations. If discrepancy principle $\left\|y-A x_{k^{*}}\right\| \leq \gamma \varepsilon$ is satisfied, exit with current iteration $x_{k^{*}}$ as solution.

6. Perform Padé iteration steps $x_{i+1}=P^{(2,1)} x_{i}$, (not projected!) until discrepancy principle is satisfied. $k^{*}$ is the stoppping index, $x_{k^{*}}$ our regularized solution.

Remark. If $\left(b_{i}\right)$ contains double entries, i.e. a base point has been chosen more than once, it is always possible to increase $m$ and repeat step 3 until this no longer occurs. This is more of a pathological condition though, that did not occur yet in our experiments.

Described in words, what this algorithm accomplishes is shifting the base points of the splines towards the points with larger function values in their vicinity; the vector $w$ describes a set of weights that are applied to the partial sums of the function up to a specific index. When the sum is greater than a certain portion of the total sum over the discretized function, the corresponding place will be added to the list of base points. By setting $w_{0}=r_{\min }$ and $w_{n}=r_{\max }$ respectively, we ensure that the left and right end of the interval considered are always included in the list of base points. The whole procedure of shifting the base points is done $l$ times. This, together with the projection of the spline coefficients, is the large improvement of the algorithm; using a pure Padé iteration without an adaptation of base points or projection will result in speedups, but not in noticeably better results. The adaption of base points and the projection, is also exactly what distinguishes algorithm 3 from algorithm 2.

Let us take a look at the example presented in Fig. (3). The true solution here is a monomodal log-normal distribution with the values $r_{\text {med }}=0.1, \bar{\sigma}=1.6$ and $N_{t}=1$, example 1 from Table 1 . We will consider the distribution in the interval $[0,1]$ (note here that the unit for the radius will always be $\mu \mathrm{m}$ in this paper). We start the Padé iteration with nine equidistant base points on the interval $[0,1]$. In all our pictures, the solid line denotes the true solution, the dashed line our reconstruction, and the $\times$ symbols mark the base points. One can see this very well in Fig. (3a) how the solution after one iteration step already takes a form with a peak at about the same radius as the true solution, but heavily smoothed out. In the next plot (b), the first change of base points has taken place; it is possible to see that, while the smoother parts of the reconstruction stay smooth, the peak becomes more pronounced. Observe how the base points have already moved to the left here. In plot (c), after ten iteration steps, this effect is still stronger. Plot (d) is the final iteration step with a reconstruction error of about $8 \%$. The input error on the data $y$ was $3 \%$. This is not a spectacular result, as this is one of the more unproblematic distributions for this inversion. 


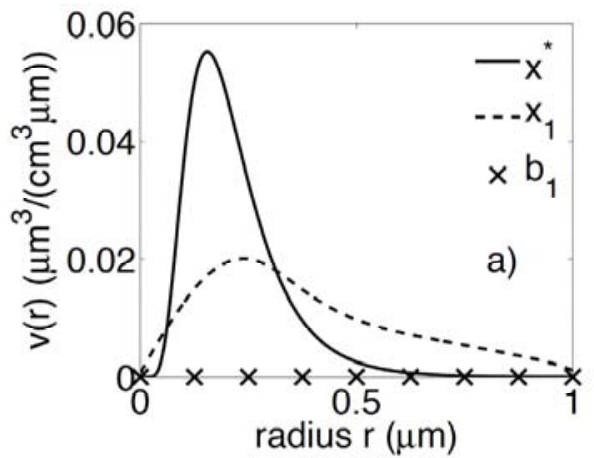

(a)

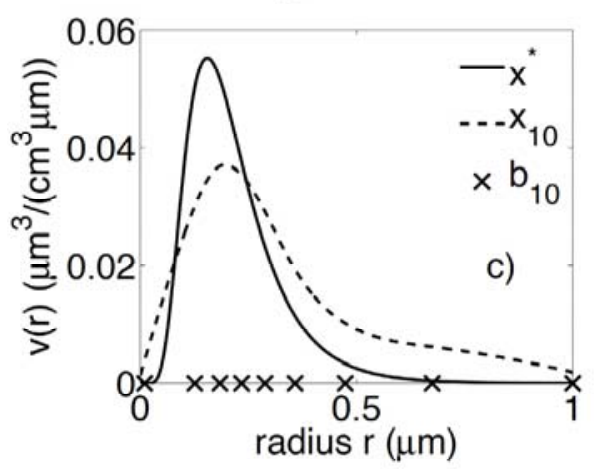

(c)

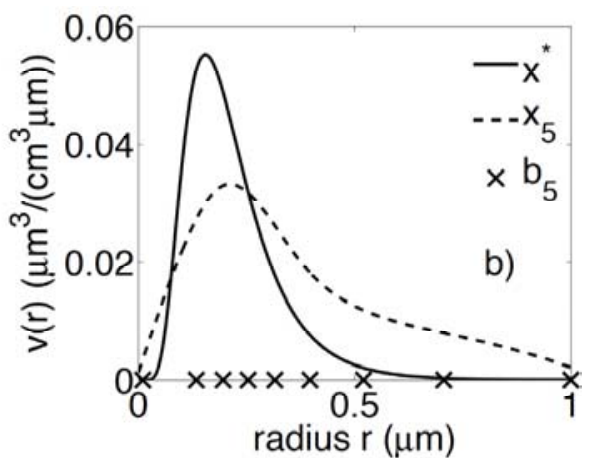

(b)

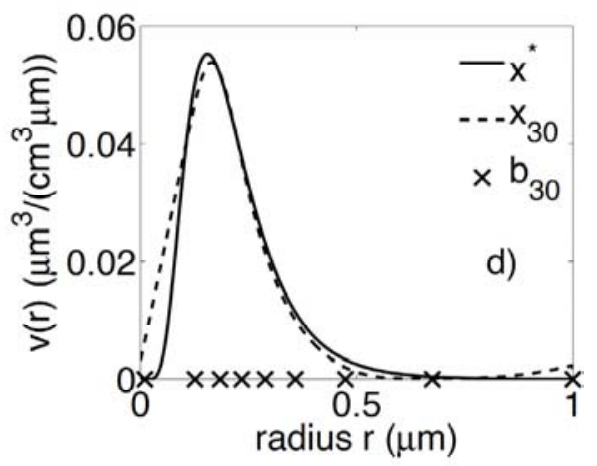

(d)

Fig. (3). Reconstruction of the distribution from Fig. (1) with algorithm 3. From the left to the right, the reconstruction is shown after 1,5 , 10 and 30 iteration steps as the dashed line. The solid line is the true solution, the $\times$ symbols mark the base points at each shown iteration. Observe how the base points adapt themselves to the structure of the true solution.

It is possible to see here that both our projection and the base point change are beneficial to the algorithm; in Fig. (1a), the projection is turned off, in (b), the base point change. The relative errors here are $30 \%$ or $18 \%$, respectively. If the solution is not projected, the distribution is marred by an artifact near the end of the distribution, where the function drops below zero. If the base points are left distributed equidistantly, the reconstructed mode will become too wide and the median radius not reconstructed properly.

We will now consider another monomodal log-normal distribution, case 2 from Table 1. This distribution is known to be quite problematic in the reconstruction, insofar as standard algorithms as in [4,6] fail to deliver sensible results here. Our algorithm seems to work very well with it, though. Look at Fig. (4) to see the reconstruction after 30 steps. the reconstruction error for an input error of $1 \%$ was less than $6 \%$.

\section{NUMERICAL RESULTS}

Now we will see how well the presented algorithm will do in lidar simulation. For all the simulation setups used, look at Table $\mathbf{1}$.

For all our test runs, we chose the spline degree $d=4$, the vector of starting base points $b_{0}$ with 9 components, the number of projected iteration steps $p=30$ (for algorithm 3), the discrepancy principle parameter $\gamma=1.1$ and the relaxation parameter $\tau=1 /\|A\|^{2}$ for algorithm 2 and $\tau=100 /\|A\|^{2}$ for algorithm 3 .

Note here that all the simulations we made have been made with a wavelength setup of $3+2$, meaning backscatter wavelengths at 355, 532 and $1064 \mathrm{~nm}$ and extinction wavelengths at 355 and $532 \mathrm{~nm}$. These are the wavelengths usually found in application setups, see for instance [14]; of

Table 1. Simulation Cases for Microphysical Retrieval. The Parameters $r_{\text {med }}$ (in $\mu \mathrm{m}$ ), $\bar{\sigma}$ and $N_{t}$ are the Ones Defining a MonoModal Log-Normal Distribution, see Equation (13), $m$ is the Complex Refractive Index

\begin{tabular}{|c|c|c|c|c|c|}
\hline Case & Number of Modes & $\boldsymbol{r}_{\text {med }}$ & $\overline{\boldsymbol{\sigma}}$ & $\boldsymbol{N}_{\boldsymbol{t}}$ & $\boldsymbol{m}$ \\
\hline \hline 1 & 1 & 0.1 & 1.6 & 1 & 1 \\
\hline 2 & 1 & 0.5 & 1.2 & $(1,0.05)$ & $1.5+0.01 \mathrm{i}$ \\
\hline 3 & 2 & $(0.1,0.5)$ & $(1.6,1.2)$ & $(0.5,0.01)$ & $1.4+0.075 \mathrm{i}$ \\
\hline 4 & 2 & $(0.1,0.5)$ & $(1.6,1.2)$ & & $1.7+0.005 \mathrm{i}$ \\
\hline
\end{tabular}


course, simulating more wavelengths may generate better results, but these experiments have been omitted because such data will usually not be available in an application scenario. We performed our simulations (also see [15] for the more general framework) for this case exclusively as it is the most common setup used that is viable for the retrieval of the volume distribution at all (for less than $3+2$ wavelengths, we do not expect any good results, see [10,5]). For the future, especially setups with more extinction wavelengths can become interesting as the Lidar data could for instance be combined with sun photometer measurements, see [19].

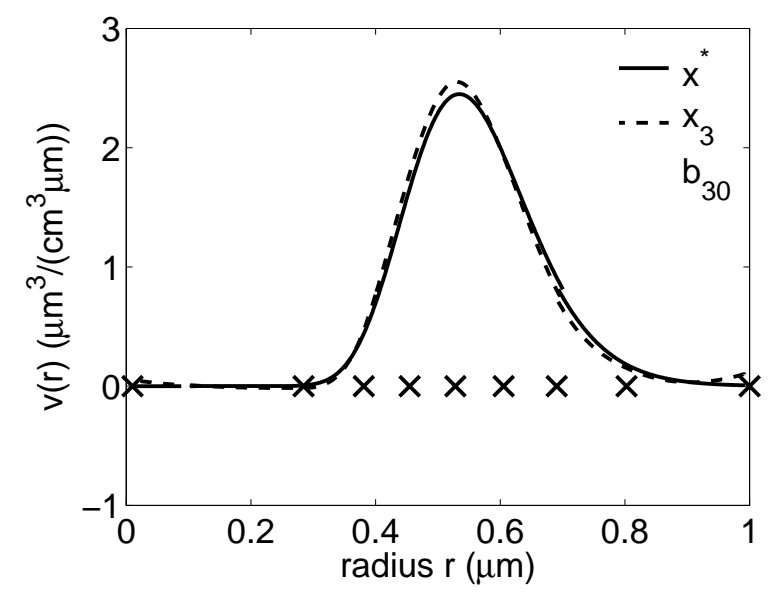

Fig. (4). Reconstruction of case 2 for $\varepsilon^{*}=1 \%$ with algorithm 3 .

Note that while all these calculations could also be done for the number density function $n(r)$ (see equation (1)), we actually perform them with the volume distribution for numerical stability reasons. The volume distribution is given by $v(r)=\frac{4 \pi r^{3}}{3} n(r)$, so number and volume concentration can be directly calculated from each other. All our examples given will be mono- or multimodal log-normal distributions, defined already in equation (13). We will work with the associated volume distribution accordingly. All the data perturbations we perform will be Gaussian random, fulfilling the property $\frac{\left\|y-y^{*}\right\|}{\left\|y^{*}\right\|}=\varepsilon^{*}$, so $\varepsilon^{*}$ denotes the noise level in percent.

Throughout the paper, we will do simulations on four different noise levels: $1 \%, 5 \%, 15 \%$ and $25 \%$. The perturbed signals are gained by applying a Gaussian error to the data and normalizing the error so that $\frac{\left\|y_{\varepsilon}-y\right\|}{\|y\|}=\varepsilon^{*}$ is satisfied. For now, we will compare the quality of the reconstruction for the effective radius of the particles; this is derived from the particle surface-area concentration (which has the unit $\mu \mathrm{m}^{2} \mathrm{~cm}^{-3}$ ),

$a_{t}=3 \int \frac{v(r)}{r} \mathrm{~d} r$,

and the volume concentration (which has the unit $\mu \mathrm{m}^{3} \mathrm{~cm}^{-3}$ ), $v_{t}=\int v(r) \mathrm{d} r$

and is defined by

$r_{\text {eff }}=3 \frac{v_{t}}{a_{t}}$.

Later on, we will also try to retrieve the complex refractive index of the particles, see section 6 .

Now, let us take a look at the results in Tables $\mathbf{2}$ and $\mathbf{3}$. For all four cases, the effective radius has been retrieved 100 times for each of the four different error levels. That means that a set of 100 different sets of perturbed data were processed by each algorithm.

Table 2. Retrieval Results for the Effective Radius for the Cases 1 and 2 from Table 1. The First Row Always Gives the mean Value and Standard Deviation of the Retrieved Effective Radius for 100 Runs, the Second Row the Median/Mean Retrieval Error in Percent. Note that the Unit for $r_{\text {eff }}$ is $\mu \mathrm{m}$

\begin{tabular}{|c|c|c|c|}
\hline Noise Level & Algorithm 1 & Algorithm 2 & Algorithm 3 \\
\hline \hline Case 1. $r_{\text {eff }}=0.17$. & \multicolumn{3}{|l|}{} \\
\hline $1 \%$ & $0.26 \pm 0.04$ & $0.13 \pm 0.003$ & $0.14 \pm 0.003$ \\
& $53 \% / 53 \%$ & $26 \% / 26 \%$ & $20 \% / 21 \%$ \\
\hline $5 \%$ & $0.19 \pm 0.055$ & $0.14 \pm 0.007$ & $0.14 \pm 0.006$ \\
& $31 \% / 26 \%$ & $22 \% / 22 \%$ & $19 \% / 19 \%$ \\
\hline $15 \%$ & $0.20 \pm 0.061$ & $0.15 \pm 0.023$ & $0.15 \pm 0.019$ \\
& $20 \% / 12 \%$ & $17 \% / 16 \%$ & $13 \% / 11 \%$ \\
\hline $25 \%$ & $0.25 \pm 0.043$ & $0.16 \pm 0.04$ & $0.17 \pm 0.033$ \\
& $48 \% / 48 \%$ & $21 \% / 22 \%$ & $17 \% / 18 \%$ \\
\hline Case 2. $r_{\text {eff }}=0.54$. & & & \\
\hline $1 \%$ & $0.28 \pm 0$ & $0.58 \pm 0.368$ & $0.63 \pm 0.211$ \\
& $50 \% / 50 \%$ & $45 \% / 35 \%$ & $28 \% / 22 \%$ \\
\hline $5 \%$ & $0.28 \pm 0$ & $1.17 \pm 0.698$ & $0.51 \pm 0.148$ \\
& $50 \% / 50 \%$ & $132 \% / 103 \%$ & $20 \% / 12 \%$ \\
\hline $5 \%$ & $0.28 \pm 0$ & $1.28 \pm 1.14$ & $0.48 \pm 0.067$ \\
& $50 \% / 50 \%$ & $146 \% / 82 \%$ & $12 \% / 15 \%$ \\
\hline & $0.32 \pm 0.09$ & $0.64 \pm 4.767$ & $0.40 \pm 0.323$ \\
& $42 \% / 42 \%$ & $230 \% / 53 \%$ & $41 \% / 33 \%$ \\
\hline
\end{tabular}

Case 1 is a standard example that most algorithms for the retrieval of microphysical parameters have little trouble with. Indeed, this can be observed here too. One can see algorithm 1 struggling here, as small error levels have the interesting effect of completely invalidating the results. For higher error levels, algorithm 1 becomes better suited. While the Padé algorithms seem to have a slight problem about always underestimating the effective radius (which could be produced by the discrepancy principle stopping too early, which is a common problem for low error levels), the hybrid 
method (algorithm 1) overestimates. Also, the higher the error level gets, the more pronounced the difference between the projected algorithm 3 and the unprojected algorithms 1 and 2. Note that sometimes, solution seem to be better for higher error levels; this could be attributed to the fact that the solution for errorless data already has some error (due to the ill-posedness), while higher errors have a tendency towards randomizing the results more, away from the solution for errorless data.

Table 3. Retrieval Results for the Effective Radius for the Cases 3 and 4 from Table 1. The First Row Always Gives the Mean Value and Standard Deviation of the Retrieved Effective Radius for 100 Runs, the Second Row the Median/Mean Retrieval Error in Percent. Note that the Unit for $r_{\text {eff }}$ is $\mu \mathrm{m}$

\begin{tabular}{|c|c|c|c|}
\hline Noise Level & Algorithm 1 & Algorithm 2 & Algorithm 3 \\
\hline \hline Case 3. $r_{\text {eff }}=0.34$. & \multicolumn{3}{|c|}{} \\
\hline $1 \%$ & $0.37 \pm 0.002$ & $0.31 \pm 0.054$ & $0.32 \pm 0.063$ \\
& $9 \% / 9 \%$ & $15 \% / 11 \%$ & $13 \% / 9 \%$ \\
\hline $5 \%$ & $0.30 \pm 0.057$ & $0.29 \pm 0.034$ & $0.29 \pm 0.04$ \\
& $22 \% / 20 \%$ & $17 \% / 17 \%$ & $17 \% / 17 \%$ \\
\hline $15 \%$ & $0.28 \pm 0.038$ & $0.29 \pm 0.273$ & $0.30 \pm 0.046$ \\
& $20 \% / 18 \%$ & $41 \% / 28 \%$ & $15 \% / 14 \%$ \\
\hline $25 \%$ & $0.20 \pm 0.035$ & $0.24 \pm 0.219$ & $0.26 \pm 0.054$ \\
& $41 \% / 41 \%$ & $33 \% / 33 \%$ & $23 \% / 21 \%$ \\
\hline Case 4. $r_{\text {eff }}=0.27$. & & & \\
\hline $1 \%$ & $0.23 \pm 0.003$ & $0.21 \pm 0.015$ & $0.21 \pm 0.018$ \\
& $15 \% / 15 \%$ & $22 \% / 21 \%$ & $22 \% / 21 \%$ \\
\hline $5 \%$ & $0.21 \pm 0.025$ & $0.21 \pm 0.012$ & $0.21 \pm 0.026$ \\
& $20 \% / 20 \%$ & $25 \% / 24 \%$ & $20 \% / 20 \%$ \\
\hline $5 \%$ & $0.21 \pm 0.049$ & $0.20 \pm 0.026$ & $0.21 \pm 0.020$ \\
& $28 \% / 20 \%$ & $26 \% / 26 \%$ & $20 \% / 19 \%$ \\
\hline & $0.30 \pm 0.071$ & $0.20 \pm 0.030$ & $0.21 \pm 0.029$ \\
& $40 \% / 30 \%$ & $28 \% / 27 \%$ & $21 \% / 20 \%$ \\
\hline
\end{tabular}

The situation in case 2 looks quite different. Algorithms 1 and 2 completely fail here. Algorithm 2 has big problems because of the "empty space" before the mode, see Fig. (4) in the range from $0-0.3 \mu \mathrm{m}$; a large chunk of negative distribution is produced here (not shown). Something similar is true for algorithm 1; because this algorithm does not assume the base points to be distributed equidistantly, but by the zeros of Chebyshev polynomials, there are much more base points in the lower radius range than in the higher, resulting in a distribution which looks nothing the one to be reconstructed (not shown); also, because it is extremely smoothed out, it is nearly not possible to see any difference for the different error levels. Algorithm 3 is the only one who fares well in this example, because the projection counters the effect of large negative areas in the reconstruction.

All algorithms fare reasonably well for case 3 (see Fig. 5 for an example with algorithm 3); algorithm 2 has some outliers though, making that algorithm slightly less reliable than the others. This can mostly be seen by the high standard deviation for the higher error levels. Also, algorithm 1 seems to have slight problems at the higher error levels.

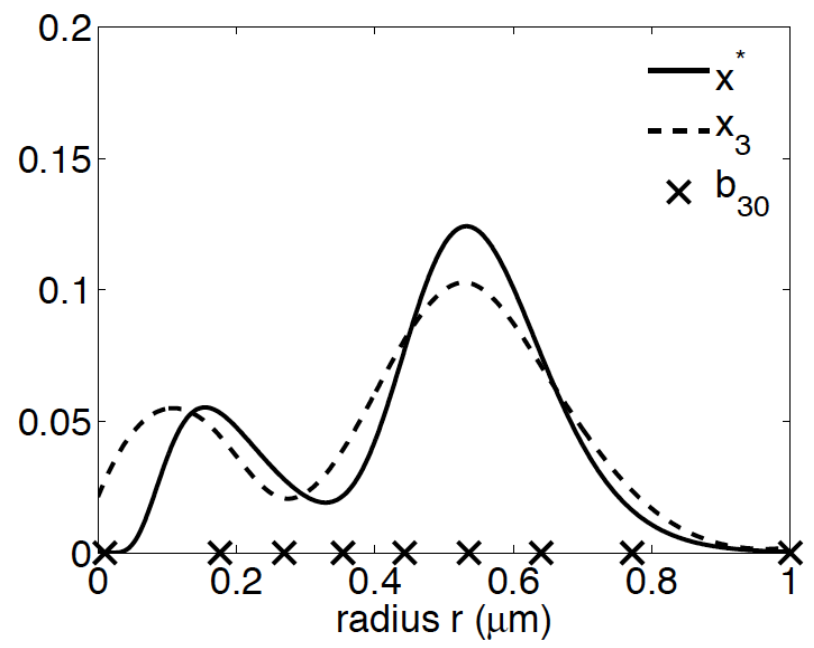

Fig. (5). Reconstruction of case 3 for $\varepsilon^{*}=1 \%$ with algorithm 3 .

Case 4 also gets reconstructed fairly well by all algorithms, with a slight advantage for algorithm 3, especially at higher error levels.

Note here that we exclusively looked at simulation examples where the maximum sensible radius for the size of the particles can be set to $1 \mu \mathrm{m}$. This is due to some restrictions. For one, our algorithm for calculating the kernel function is based on the one found in [2], which works in a recursive way and requires many more calculations for large radii; thus, integrating over this implementation of the kernel needs a lot more calculation time if the maximum radius is set to a high value. Much more important are the physical restrictions, though; as we are looking at Mie theory here, we know that we need the measuring wavelengths and the particle size in roughly similar magnitudes. Since we are looking at lidar data, we know that in practice our largest available wavelength is $1064 \mathrm{~nm}$, making much larger radii infeasible to retrieve. Of course, this does not concern the algorithm itself, but only the source data; if the lidar data could, for instance, be combined with sun photometer data at larger wavelengths, the algorithm should still provide sensible results. To test this, we experimented with a simulated mono-modal distribution with $r_{\text {med }}=0.1, \bar{\sigma}=2.5$ and $N_{t}=1$, and assumed a maximum radius of $6 \mu \mathrm{m}$. A data error of $5 \%$ was added. First, we ran the algorithm with the same five wavelengths as before (extinction and backscatter each at 355 and $532 \mathrm{~nm}$ and backscatter at $1064 \mathrm{~nm}$ ). See the result in Fig. (6). It is possible to see how the data obviously contains very little information behind a certain point, while the area with smaller radii is still reconstructed in an acceptable manner. For the second experiment, we added an extinction wavelength at $1064 \mathrm{~nm}$, backscatter and extinction wavelengths each at 1596 and $3192 \mathrm{~nm}$ and a backscatter 
wavelength at $6384 \mathrm{~nm}$. Note that these wavelengths are purely artificial, and have been chosen as to roughly approximate the spacing of the true available lidar wavelengths. See Fig. (7) for the result. The algorithm was able to perform a good reconstruction up to a radius of $6 \mu \mathrm{m}$.

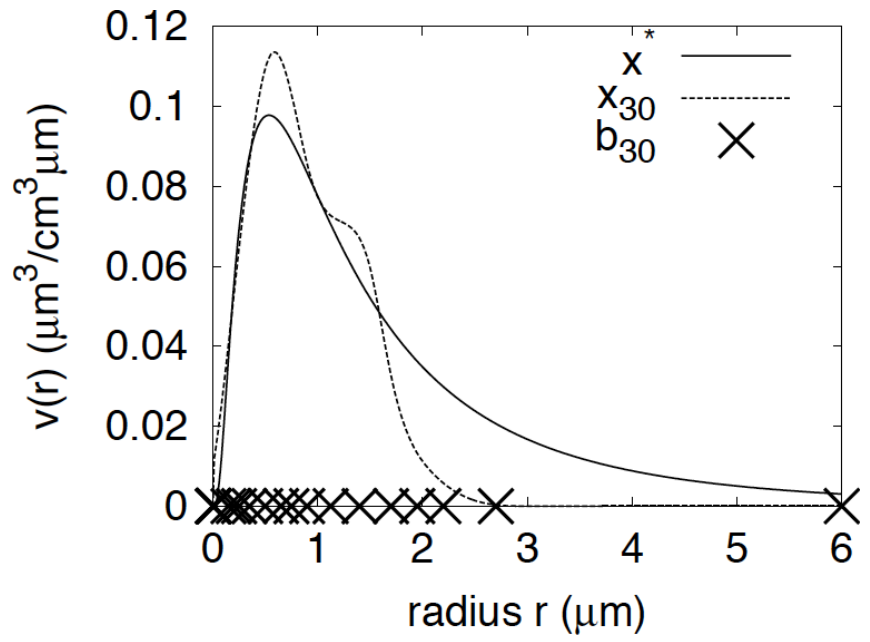

Fig. (6). Reconstruction of a very wide mode with with $r_{\text {med }}=0.1, \bar{\sigma}=2.5$ and $N_{t}=1$, for $\varepsilon^{*}=5 \%$, performed with the usual five wavelengths and algorithm 3 .

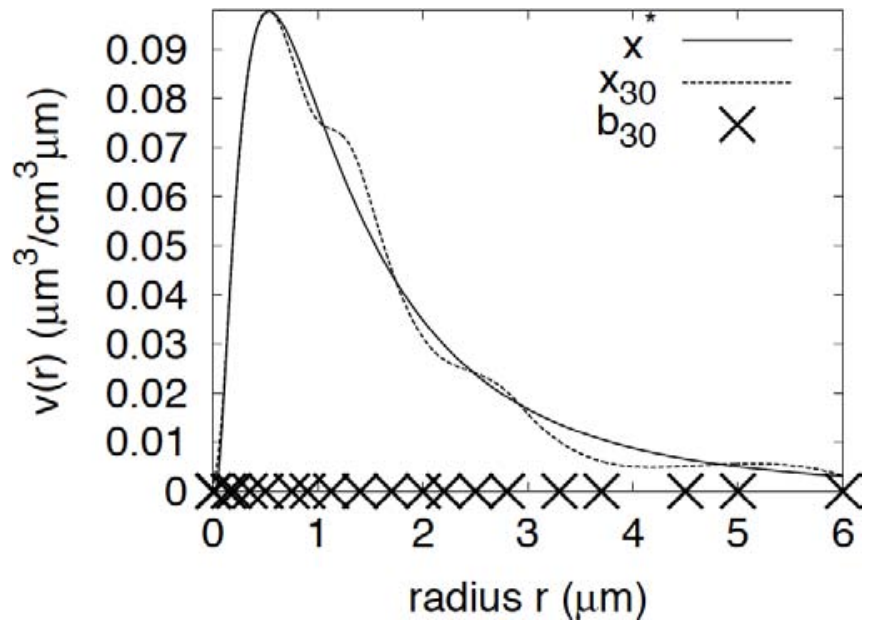

Fig. (7). Reconstruction of a very wide mode with with $r_{\text {med }}=0.1, \bar{\sigma}=2.5$ and $N_{t}=1$, for $\varepsilon^{*}=5 \%$, performed with the usual five wavelengths plus an extinction wavelength at $1064 \mathrm{~nm}$, backscatter and extinction wavelengths each at 1596 and $3192 \mathrm{~nm}$ and a backscatter wavelength at $6384 \mathrm{~nm}$ and algorithm 3 .

\section{UNKNOWN REFRACTIVE INDEX}

Now, the question remains how to retrieve the complex refractive index $m$ in the case it is not known, which will be the usual case in an application scenario. Note here that this has not been done yet with a Padé iteration. This is usually solved by breaking down the nonlinear problem into a finite set of linear problems, by separately calculating solutions for the problem at every point of a discretized grid of refractive indices. This has been done in [4], for instance. Note that the procedure used there fails here; while the algorithm from [4] calculates a certain set of different solutions at every point of the refractive index grid and chooses the one with the smallest residual (in other words, $\min \|y-A x\|$, with the $x$ being from the set of possible results mentioned earlier), the algorithms here calculate only one solution per refractive index; furthermore, since we are using a true regularization algorithm stopped by the discrepancy principle, it is obvious that using the grid of residuals $\|y-A x\|$ cannot lead to sensible results. Because the discrepancy stops the iteration at the step where $\left\|y-A x_{k^{*}}\right\| \leq \gamma \varepsilon$ holds, the retrieved residual will be nearly identical for all refractive indices, rendering that criterion worthless in this case. So, our approach must be slightly altered here; instead of using the discrepancy principle, we stop the iteration after a fixed number of iterations that is determined in some way by the input error level, thus ensuring that over- and underregularization is restricted. Experiments have shown us that the very simple formula

$$
k^{*}=\left\lfloor\left(\varepsilon^{*}\right)^{-1}\right\rfloor
$$

is sufficient for that, where the $\left\lfloor\left(\varepsilon^{*}\right)^{-1}\right\rfloor$ operator denotes the integer part of the real number $\left(\varepsilon^{*}\right)^{-1}$.

This procedure has led to some very interesting results. The grid used was always the same, 21 equidistant points on the real axis from 1.3 to 1.8 and 21 points on the imaginary axis from 0 to 0.1 .

Table 4. Simulation Cases for Refractive Index Retrieval. The Parameters $r_{\text {med }}$ (in $\mu \mathrm{m}$ ), $\bar{\sigma}$ and $N_{t}$ are the Ones Defining a Mono-Modal Log-Normal Distribution, see Equation (13), $m$ is the Complex Refractive Index

\begin{tabular}{|c|c|c|c|c|}
\hline Case & $\boldsymbol{r}_{\text {med }}$ & $\overline{\boldsymbol{\sigma}}$ & $\boldsymbol{N}_{\boldsymbol{t}}$ & $\boldsymbol{m}$ \\
\hline \hline 1 & 0.1 & 1.6 & 1 & $1.4+0.075 \mathrm{i}$ \\
\hline 2 & 0.5 & 1.2 & 1 & $1.7+0.005 \mathrm{i}$ \\
\hline
\end{tabular}

Experiments have shown (see [4]) that we expect to see a diagonal structure in the retrieved refractive index grid, ideally with the point of lowest residual error on the diagonal and close to the true solution. See Table $\mathbf{4}$ for the two test cases examined here. Generally, retrieval works better in cases where the ill-posedness of the problem is smallest for the true solution, i.e. in cases with high real and low imaginary part of the refractive index, meaning case 2 in our experiment cases, see Fig. (8). The diagonal is present and very sharply defined, making it easy to pick out a suitable refractive index as solution. For more ill-posed problems, like case 1 , retrieval of the refractive index is much less stable and more prone to error, see Fig. (9). The diagonals here get noticeably larger, needing human interaction to pick out suitable refractive indices. It is possible to see that the other algorithms are prone to picking a "best solution" somewhere on the border of the examined grid.

The results of the refractive index retrieval have been summarized in Table 5. Note that the solution with the smallest residual as reconstructed refractive index was automatically picked. For every error level, example and algorithm, we performed 100 test runs. We always use $21 \times 21$ grids for the refractive index, which according to 


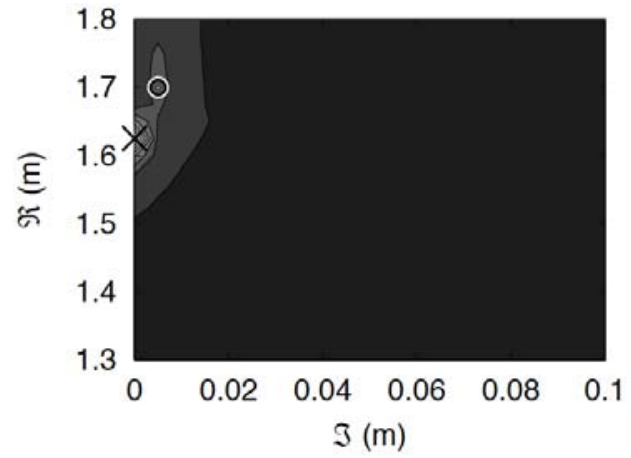

(a)

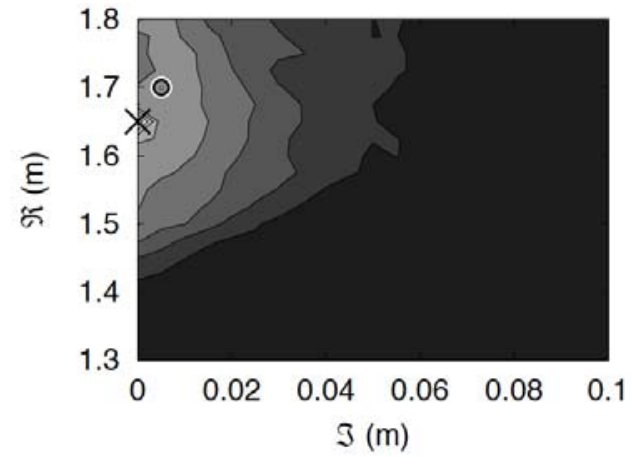

(b)

Fig. (8). Refractive index grids for case 2 from Table 4 with a data error of 5\% for algorithms $2((\mathbf{a})$ on the left) and 3 ((b) on the right). The circle denotes the true refractive index, the $\times$ mark the retrieved one. Also $\mathfrak{R}(m)$ denotes the real part and $\mathfrak{I}(m)$ denotes the imaginary part of the complex number $m$.

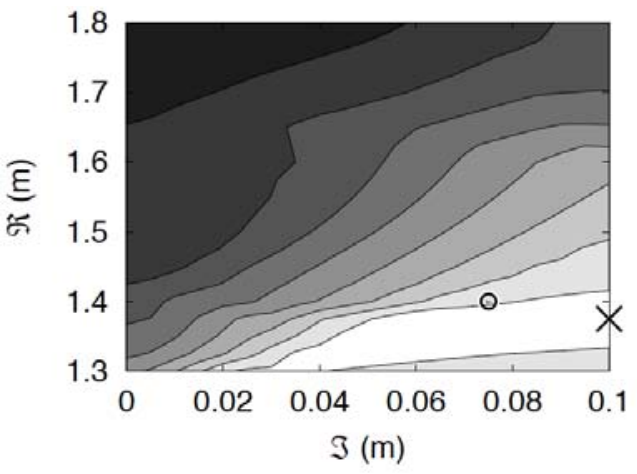

(a)

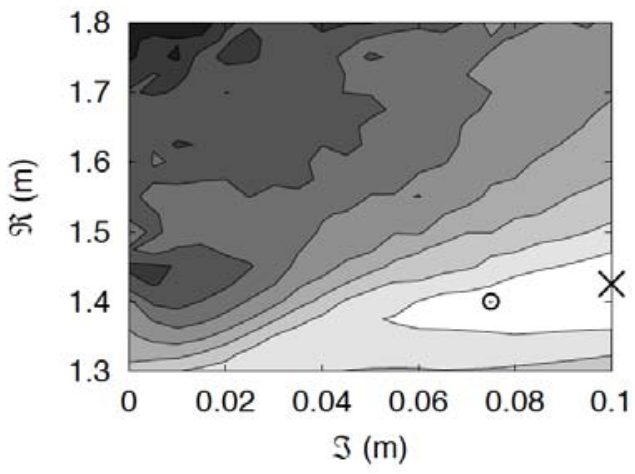

(b)

Fig. (9). Refractive index grids for case 1 from Table 4 with a data error of 5\% for algorithms 2 ((a) on the left) and 3 ((b) on the right).

computational experiments (see [13]) is a good compromise between speed and accuracy for the retrieval of the refractive index (on a normal workstation PC, one run needs about 15 minutes to complete).

Now, let us take a look at Table 5. For the mean value of a vector $n_{1}, \ldots, n_{k}$ we assume the usual definition $\bar{n}=\frac{1}{k} \sum_{j=1}^{k} n_{j}$. Also, if we assume the values are sorted like $n_{1} \leq n_{2} \leq \ldots \leq n_{k}$, the median value is defined by $\tilde{n}=n_{(k+1) / 2}$ for odd $k$ and $\tilde{n}=\frac{n_{k / 2}+n_{(k+1) / 2}}{2}$ for even $k$. One can see that the quality of the reconstruction not only depends on the algorithm, but also on the example. For the two examples, which are very different in nature (low real part and high imaginary part of the refractive index means a very high degree of ill-posedness, the opposite a lower degree), one can see that the quality of the results differ greatly. While all algorithm are able to produce acceptable results for case 2, case 1 is much more tricky. Both examples here get handled best by the new algorithm 3, though.

Also note that in contrast to previous algorithms, the numbers in Table $\mathbf{5}$ are generated automatically - no user interaction whatsoever was done to obtain these results. This might get us a big step closer to the goal of fully-automated lidar data processing as outlined in [15].
Now, keep in mind that one of the reasons we try to retrieve the refractive index is because we are trying to discern the particles present in the examined layer. A big problem here is distinguishing the different kinds of particles that have very low imaginary parts of the refractive index, about $\mathfrak{I}(m)<0.01$. To see how well our algorithms can cope with that problem, we perform another simulation

experiment. We only try to retrieve the imaginary part of the refractive index now, and sort them into different bins. We propose to define the bins between the imaginary values 0 , $0.001 \mathrm{i}, 0.005 \mathrm{i}, 0.0075 \mathrm{i}$ and $0.01 \mathrm{i}$. Then we take the monomodal distribution from case 2 from Table $\mathbf{4}$, and assume different refractive indices of $m_{j}=1.5+q_{j}$, where

$q=(0,0.0005,0.002,0.004,0.006,0.009)$

and see if they are sorted into the correct bins by the different algorithms. Again, this is done a hundred times per error level, with algorithm 3 . The grid used was $21 \times 21$ points, but with a maximum imaginary part of $\mathfrak{I}(m)=0.01$. See Table 6 for results. One can see that distinguishing the imaginary part with the help of the bins leads to satisfactory results. The accuracy for all cases lies in similar dimensions as the input error level, which is a good result. Thus, this algorithm might also be suited, maybe with some future extensions and modifications, to accurately distinguish between particles with a low imaginary refractive index. 
Table 5. This Table Shows the Retrieval Results for the Refractive Index for Cases 1 and 2 with Algorithms 1-3. Displayed is the Median Reconstruction Error (Real Part/Imaginary Part. Real and Imaginary Parts were Examined Separately for the Median, but they are Written in One Line for Clarity)

\begin{tabular}{|c|c|c|c|}
\hline Noise Level & Algorithm 1 & Algorithm 2 & Algorithm 3 \\
\hline \hline Case 1. $m=1.4+0.075$ i. \\
\hline $1 \%$ & $0.1 / 0.02 \mathrm{i}$ & $0.1 / 0.015 \mathrm{i}$ & $0.1 / 0.015 \mathrm{i}$ \\
\hline $5 \%$ & $0.15 / 0.04 \mathrm{i}$ & $0.125 / 0.025 \mathrm{i}$ & $0.15 / 0.025 \mathrm{i}$ \\
\hline $15 \%$ & $0.15 / 0.04 \mathrm{i}$ & $0.175 / 0.03 \mathrm{i}$ & $0.175 / 0.025 \mathrm{i}$ \\
\hline $25 \%$ & $0.2 / 0.05 \mathrm{i}$ & $0.225 / 0.035 \mathrm{i}$ & $0.175 / 0.03 \mathrm{i}$ \\
\hline Case 2. $m=1.7+0.005 \mathrm{i}$. & & \\
\hline $1 \%$ & $0.05 / 0.005 \mathrm{i}$ & $0.05 / 0$ & $0.05 / 0$ \\
\hline $5 \%$ & $0.05 / 0.005 \mathrm{i}$ & $0.05 / 0$ & $0.05 / 0$ \\
\hline $15 \%$ & $0.05 / 0.01 \mathrm{i}$ & $0.05 / 0.005 \mathrm{i}$ & $0.05 / 0.005 \mathrm{i}$ \\
\hline $25 \%$ & $0.075 / 0.015 \mathrm{i}$ & $0.1 / 0.015 \mathrm{i}$ & $0.075 / 0.01 \mathrm{i}$ \\
\hline
\end{tabular}

\section{APPLICATION CASE STUDY}

To validate our algorithm, we applied it to real multiwavelength Raman lidar data for a specific height above the Planetary Boundary Layer (i.e. between 2000 and $2500 \mathrm{~m}$ ). The measurements were taken during a biomass burning event that occurred on June 28-30, 2007, over Athens, Greece.

In this paper, the lidar measurements taken on June 29 , 2007 are examined. At this date, the smoke was still quite fresh (about 1-1.5 hours old), so it is expected for the aerosols to be very weakly absorbing (see [20,21]). Also, concurrent radiosonde data of the relative humidity gave a value of the order of $50 \%$ and a potential temperature of 285 $\mathrm{K}$. Then, we used our algorithm to retrieve both the volume distribution and the refractive index of the aerosols (see Figs. (10, 11 for details); as there was no available information of the error level, we fixed the iteration to 30 steps. As can be expected, the diagonal in the refractive index grid is clearly visible in Fig. (10), with a minimum residual error at $m=1.37$ and an average index for the ten best points of $m=1.386+0.006 \mathrm{i}$. The refractive index calculated by our

Table 6. Results for Distinguishing Particles of Low Imaginary Refractive Index. The Real Part of the True Refractive Index was 1.5. The Percentages Say how Often the Refractive Index was Put in the Respective Bin. Each Column Marks a Specific Bin, with the Lower and Upper Bound Given in the Column Header. The Asterisk Symbols Mark the Correct Bin

\begin{tabular}{|c|c|c|c|c|}
\hline Noise Level & $0-0.001$ & $0.001-0.005$ & $0.005-0.0075$ & $0.0075-0.01$ \\
\hline \multicolumn{5}{|c|}{$\mathfrak{I}(m)=0.0005$} \\
\hline $5 \%$ & $* 77 \%$ & $23 \%$ & $0 \%$ & $0 \%$ \\
\hline $15 \%$ & $* 61 \%$ & $39 \%$ & $0 \%$ & $0 \%$ \\
\hline $1 \%$ & $0 \%$ & $* 100 \%$ & $0 \%$ & $0 \%$ \\
\hline $5 \%$ & $11 \%$ & $* 89 \%$ & $0 \%$ & $0 \%$ \\
\hline $15 \%$ & $28 \%$ & $* 69 \%$ & $3 \%$ & $3 \%$ \\
\hline $25 \%$ & $43 \%$ & $* 57 \%$ & $0 \%$ & $0 \%$ \\
\hline $15 \%$ & $6 \%$ & $* 71 \%$ & $22 \%$ & $1 \%$ \\
\hline $25 \%$ & $17 \%$ & $* 47 \%$ & $33 \%$ & $3 \%$ \\
\hline \multicolumn{5}{|c|}{$\mathfrak{I}(m)=0.006$} \\
\hline $1 \%$ & $0 \%$ & $4 \%$ & $* 93 \%$ & $3 \%$ \\
\hline $5 \%$ & $0 \%$ & $16 \%$ & $* 77 \%$ & $7 \%$ \\
\hline $15 \%$ & $1 \%$ & $20 \%$ & $* 56 \%$ & $23 \%$ \\
\hline $25 \%$ & $3 \%$ & $21 \%$ & $* 41 \%$ & $35 \%$ \\
\hline \multicolumn{5}{|c|}{$\mathfrak{I}(m)=0.009$} \\
\hline $1 \%$ & $0 \%$ & $0 \%$ & $4 \%$ & $* 96 \%$ \\
\hline
\end{tabular}


algorithm is in accordance with the values given in [20] and the references therein.

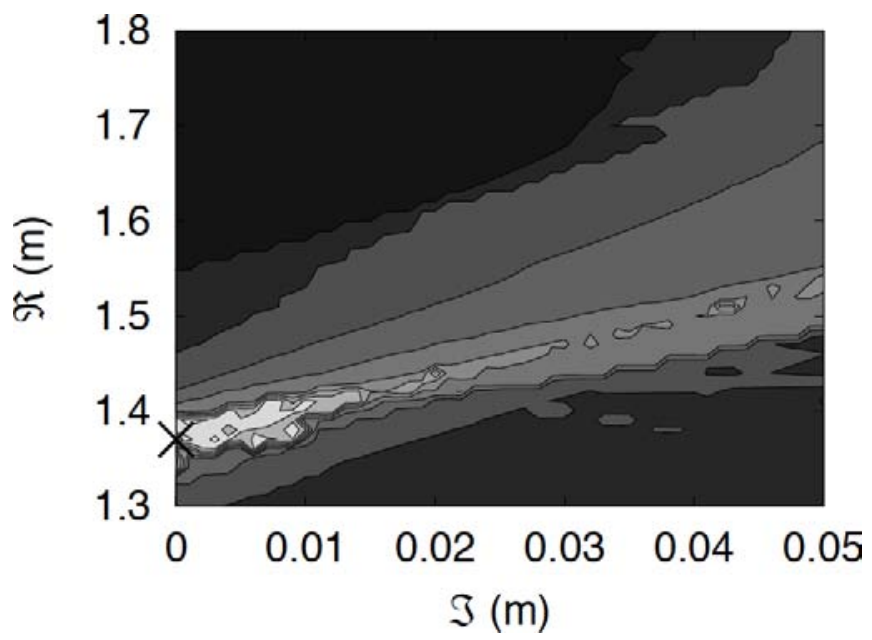

Fig. (10). Refractive index grid for the measurement data from June 29, 2007 from Athens. A diagonal line is clearly visible. The minimum refractive index can be found at the value $m=1.37$, while the proposed solution consisting of the average of the ten best points is $m=1.386+0.006 \mathrm{i}$.

We can see in Fig. (11) that the aerosol volume distribution that has been retrieved is bimodal (modes centered around 0.06 and $0.33 \mu \mathrm{m}$ in radius, respectively). This is plausible, since it is expected that the fresh smoke (larger) aerosols are quickly mixed with anthropogenic (smaller) ones emitted by various sources over the city of Athens, resulting in a mixture of quite fresh smoke aerosols and anthropogenically polluted ones. This effect could explain the bi-modal particle volume distribution found in our case. Indeed, in situ measurements of quite fresh biomass burning aerosols during the DABEX experiment in West Africa (see [21]) showed that this type of particles followed a nearly bi-modal size distribution and had preponderant radii of particles of the order of $0.051-0.35 \mu \mathrm{m}$, while similar and slightly larger values, including the aging of particles, were found in [20] and [22] over Germany during European and Canadian forest fires. However, it has to be noted that the latter paper [22] tackled the effect of mixing anthropogenic pollution and biomass burning particles, where again bi-modal particle size distributions were found. Therefore, our results on the volume distribution (and thus, the size distribution) and the range of the particle radii are in agreement with previous observations of mixing of biomass burning and anthropogenic particles.

As a next step, using as an input the measured value of the relative humidity and temperature of the atmosphere into the chemical thermodynamic model ISORROPIA II (see [23]), we inferred, after an iteration procedure, the most probable chemical composition of the sampled aerosols: $2 \%$ black carbon (BC) and 98\% organic carbon (OC) for the bigger particles (radii between 0.15 and $0.6 \mu \mathrm{m}$ ), and $1 \%$ BC, $49 \%$ ammonium sulfate and 50\% water for the smaller particles (radii between 0.01 and $0.15 \mu \mathrm{m}$ ) sampled. This corroborates our hypothesis about mixing of biomass burning and anthropogenic particles over the city of Athens.
In addition, ISORROPIA II calculated the most probable values of the refractive index of the aerosols: $m=1.4059+0.006 \mathrm{i}$ for the larger particles and $m=1.4062+0.006 \mathrm{i}$ for the smaller particles. This is in very good agreement with the retrieved refractive index of $m=1.386+0.006 \mathrm{i}$ inferred by our proposed algorithm. It is worth mentioning here that there is a need for the accurate retrieval of the imaginary part of the aerosols, as it is sometimes very hard to distinguish particles of low refractive index with $\mathfrak{I}(m)<0.01$, as stated before.

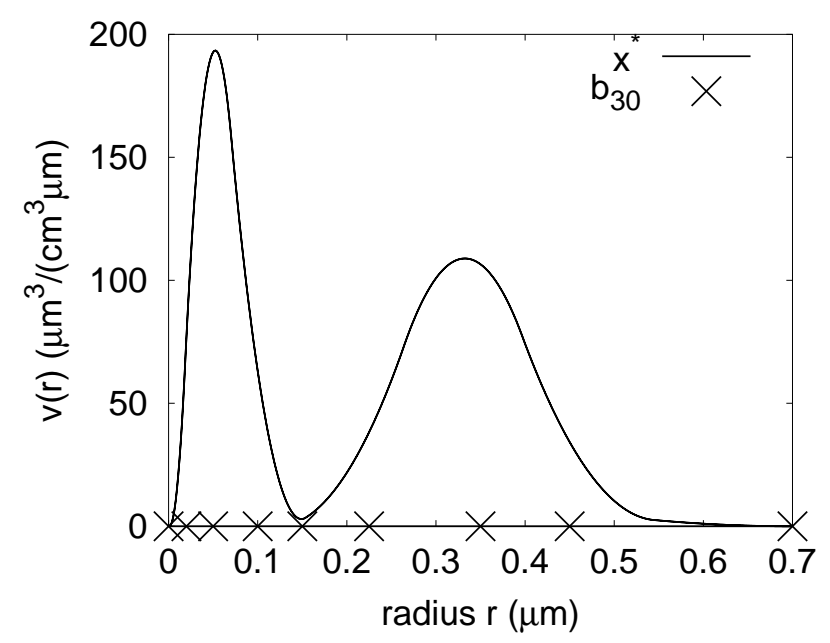

Fig. (11). The reconstructed distribution for the measurement data from June 29, 2007 from Athens for the refractive index at $m=1.37$. Note here that there are clearly two modes, a fine and a coarse one. Our experiments suggest that while these have a quite different chemical composition, the refractive indices are very similar, making the retrieval possible at this point.

Thus, our retrieved refractive index data by both the mathematical algorithm and the ISORROPIA II model are in good agreement with values reported by several authors for mixing of biomass burning with anthropogenic particles (see $[20,22])$ where the complex refractive indices ranged from 1.37 up to 1.6 (real part) and the mean imaginary part was always $<0.01$ i.

\section{CONCLUSION}

A new algorithm for the retrieval of the volume distribution and/or effective radius of aerosol layers from lidar data was presented and tested for its viability. Additionally, the possiblity of retrieving the complex refractive indices via this algorithm have been investigated. Indeed, as already shown in [10], by using Padé iteration the computer runtime can be drastically reduced. Moreover, our experiments so far prove that by choosing a suitable spline basis for the reconstruction space, better convergence results can be obtained. Also, by using the discrepancy principle for stopping, the method we presented becomes a regularization in the mathematical sense, incorporating the starting value and choice of base points as a priori information on the solution; this is also made possible by the fact that projection at the start of the iteration seems to make projection at the end unnecessary. Retrieval of the complex refractive index produces good results. Indeed, in our experiments it was possible in most cases to retrieve the refractive index fully 
automatically. Also, we have examined in how far it is possible to also distinguish between particles with small imaginary refractive indices, with promising results.

More validations, maybe with in situ measurements, will have to be performed to further assess the quality of the algorithm. Additionally, applicability of the algorithm to non-spherical particles needs to be examined.

Also, there remains the restriction that the algorithm can only retrieve one refractive index for one layer; this is problematic in cases with different aerosols with distinct refractive indices being present. Further research will be done on how to lift or work around that restriction. Moreover, determination of the regularization parameter, i.e. the number of iterations, for cases where the error level is unknown has to be furhter investigated.

\section{ACKNOWLEDGEMENT}

This work was supported by the European Commission under grant RICA-025991 via project Earlinet-ASOS which is gratefully acknowledged. Prof. A. Nenes and Dr. C. Fountoukis are acknowledged for the provision of the ISORROPIA II model.

\section{REFERENCES}

[1] Solomon S, Qin D, Manning M, et al. Climate change 2007. the physical science basis. Geneva: Switzerland, IPCC 2007.

[2] Bohren CF, Huffman DR. Absorption and Scattering of Light by Small Particles; Wiley: New York, USA 1983.

[3] Engl HW, Hanke M, Neubauer A. Regularization of Inverse Problems; Kluwer: Dordrecht, The Netherlands 2000.

[4] Böckmann C. Hybrid regularization method for the ill-posed inversion of multiwavelength lidar data to determine aerosol size distribution. Appl Opt 2001; 40:1329-42.

[5] Böckmann C, Mironova I, Müller D, Schneidenbach L, Nessler R. Microphysical aerosol parameters from multiwavelength lidar. J Opt Soc Am A 2005; 22: 518-28.

[6] Kolgotin A, Müller D. Theory of inversion with two-dimensional regularization: profiles of microphysical particle properties derived from multiwavelength lidar measurements. Appl Opt 2008; 47: 4472-90.

[7] Veselovskii I, Kolgotin A, Griaznov V, Müller D, Franke K, Whiteman D. Inversion of multiwavelength raman lidar data for retrieval of bimodal aerosol size distribution. Appl Opt 2004; 43:1180-95
Wang Y, Fan S, Feng X. Retrieval of the aerosol particle size distribution function by incorporating a priori information. J Aerosol Sci 2007; 38: 885-901.

[9] Wang Y. An efficient gradient method for maximum entropy regularizing retrieval of atmospheric aerosol particle size distribution function. J Aerosol Sci 2008; 39: 305-22.

[10] Böckmann C, Kirsche A. Iterative regularization method for lidar remote sensing. Comput Phys Commun 2006; 174: 607-15.

[11] Pappalardo G, Amodeo A, Pandolfi M, et al. Aerosol lidar intercomparison in the framework of the EARLINET project. 3 raman lidar algorithm for aerosol extinction, backscatter and lidar ratio. Appl Opt 2004; 43: 5370-85.

[12] Pornsawad P, Böckmann C, Ritter C, Rafler M. The ill-posed retrieval of aerosol extinction coefficient profiles from raman lidar by regularization. Appl Opt 2008; 47:1649-61.

[13] Osterloh L, Pérez C, Böhme D, et al. Parallel software for retrieval of aerosol distribution from lidar data in the framework of EARLINET-ASOS. Comput Phys Comm 2009; 180: 2095-102.

[14] Pappalardo G, Bösenberg J, Amodeo A, et al. EARLINET-ASOS: programs and perspectives for the aerosol study on continental scale. Proc SPIE 2006; 6367: p. 636701.

[15] Amodeo A, Mattis I, Böckmann C, et al. Optimization of lidar data processing: a goal of the EARLINET-ASOS project. Proc SPIE 2007; 1-8: 67500F.

[16] Kirsche A, Böckmann C. Padé iteration method for regularization. Appl Math Comput 2006: 180: 648-63.

[17] Rieder A. Runge-Kutta integrators yield optimal regularization schemes. Inverse Problems 2005; 21: 453-71.

[18] Deuflhard P, Hohmann A. Numerical Analysis in Modern Scientific Computing: an introduction; Springer: New York, USA 2003.

[19] Tesche M, Müller D, Ansmann A, Hu M, Zhang Y. Retrieval of microphysical properties of aerosol particles from one-wavelength raman lidar and multiwavelength sun photometer observations. Atmos Env 2008; 42: 6398-404.

[20] Müller D, Mattis I, Wandinger U, Ansmann A, Althausen D, Stoh A. Raman lidar observations of aged Siberian and Canadian forest fire smoke in the free troposphere over Germany in 2003: microphysical particle characterization. J Geophys Res 2005; 110: D17201.

[21] Johnson T, Osborne SR, Haywood JM, Harrison MAJ. Aircraft measurements of biomass burning aerosol over West Africa during DABEX. J Geophys Res 2008; 113: D00C06.

[22] Wandinger U, Müller D, Böckmann C, et al. Optical and microphysical characterization of biomass-burning and industrialpollution aerosols from multiwavelength lidar and aircraft measurements. J Geophys Res 2002; 107(D21): 8125.

[23] Fountoukis C, Nenes A. ISORROPIA II: a computationally efficient aerosol thermodynamic equilibrium model for $\mathrm{K}^{+}, \mathrm{Ca}^{+2}$ $\mathrm{Mg}^{+2}, \mathrm{NH}^{+4}, \mathrm{Na}^{+}, \mathrm{SO}_{4}^{-2}, \mathrm{NO}^{-3}, \mathrm{Cl}-, \mathrm{H}_{2} \mathrm{O}$ aerosols. Atmos Chem Phys 2007; 7 : 4639-59. 